\title{
MIRIZZI SyNDROME TyPe IV: A CHALLENGING DIAGNOSIS
}

Clin Biomed Res. 2016;36(1):44-46

1 Department of General Surgery, Hospital São Vicente de Paulo (HSVP). Passo Fundo, RS, Brazil.

2 Medical School, Universidade de Passo Fundo (UPF). Passo Fundo, RS, Brazil.

3 General Surgery Service, Hospital São Vicente de Paulo (HSVP). Passo Fundo, RS, Brazil.

4 Radiology Service, Hospital São Vicente de Paulo (HSVP). Passo Fundo, RS, Brazil.

Corresponding author: Daniel Navarini danielnavarini@hotmail.com Universidade de Passo Fundo Rua Teixeira Soares, 817. 99010-080, Passo Fundo, RS, Brazil.

\author{
Daniel Navarini ${ }^{1,2}$, Carlos Augusto Scussel Madalosso', Diego Reffatti ${ }^{1}$, \\ Luma Guareschi ${ }^{3}$, Ana Paula Schmitt ${ }^{3}$, Guilherme Marx ${ }^{3}$, \\ Paula Jaskulski², Henrique Ribeiro ${ }^{2}$, Thalis Laydner ${ }^{4}$
}

\begin{abstract}
Mirizzi Syndrome type IV is an extremely rare condition, which is confused with the diagnosis of cholangiocarcinoma in many cases. This report describes a case of a forty-three-year old patient, who was forwarded to our department of general surgery with a high suspicion of a choledochal neoplasic lesion. During the hospitalization he was diagnosed with Mirizzi Syndrome type IV. We concisely describe the case and the literature review about this pathology.
\end{abstract}

Keywords: Mirizzi Syndrome; cholangiocarcinoma; jaundice

\section{CASE REPORT}

A forty-three-year old man was forwarded from a small town in Rio Grande Do Sul to the Department of General Surgery at Hospital São Vicente de Paulo (HSVP), presenting epigastric pain, fluctuant jaundice, itch and intentional weight loss (12 kg in one year). The patient also mentioned family history of pancreatic cancer. At admission a laboratory investigation was performed and some of the exams revealed raised values with alanine aminotransferase (ALT) of $99 \mathrm{IU} / \mathrm{L}$, aspartate aminotransferase (AST) of $77 \mathrm{IU} / \mathrm{L}$, gamma glutamyl transpeptidase (GGT) of $1.171 \mathrm{IU} / \mathrm{L}$, alkaline phosphatase of $525 \mathrm{IU} / \mathrm{L}$, and direct bilirubin was slightly high with total and indirect bilirubin in a normal range. Abdominal Ultrasonography showed diffuse and symmetric intra-hepatic biliary dilatation accompanied by dilatation of the common hepatic duct, determining a 3-cm stone in the superior common bile duct. The gallbladder wall was thickened and there was an indefinition of the infundibulum due to the proximity to the choledochal stone. It was considered the hypothesis of a neoplasic lesion suggestive of cholangiocarcinoma. In order to elucidate the case, a Magnetic cholangioresonance was requested, which showed a filling defect of the cystic duct causing dilatation of the common hepatic duct and the intrahepatic biliary ducts (figure 1). The Radiology Department of HSVP argued that the impression of the magnetic resonance was compatible with Mirizzi Syndrome Type IV. Therefore, surgery was scheduled. Initially, a videolaparoscopy was performed and a large choledochal stone was identified, converting the procedure to open surgery. The cholecystectomy was made through the fundus-cystic duct detachment and the ligation of the cystic artery. The common bile duct was dilated and there was a large stone, which did not allow the exact visualization of the cystic duct. Consequently, en bloc resection was made (figure $2 \mathrm{a}$ ) and finally an end-to-side choledocho-jejunal anastomosis was performed. The surgical specimen clearly shows where the stone was located (figure $2 \mathrm{~b}$ ). The gallbladder's anatomopathological study identified gallstones in the gallbladder, chronic cholecystitis and an inflammatory process in the common bile duct. The post-operative period of our patient was satisfactory without any adverse events and all hepatic function exams were within a normal range. 


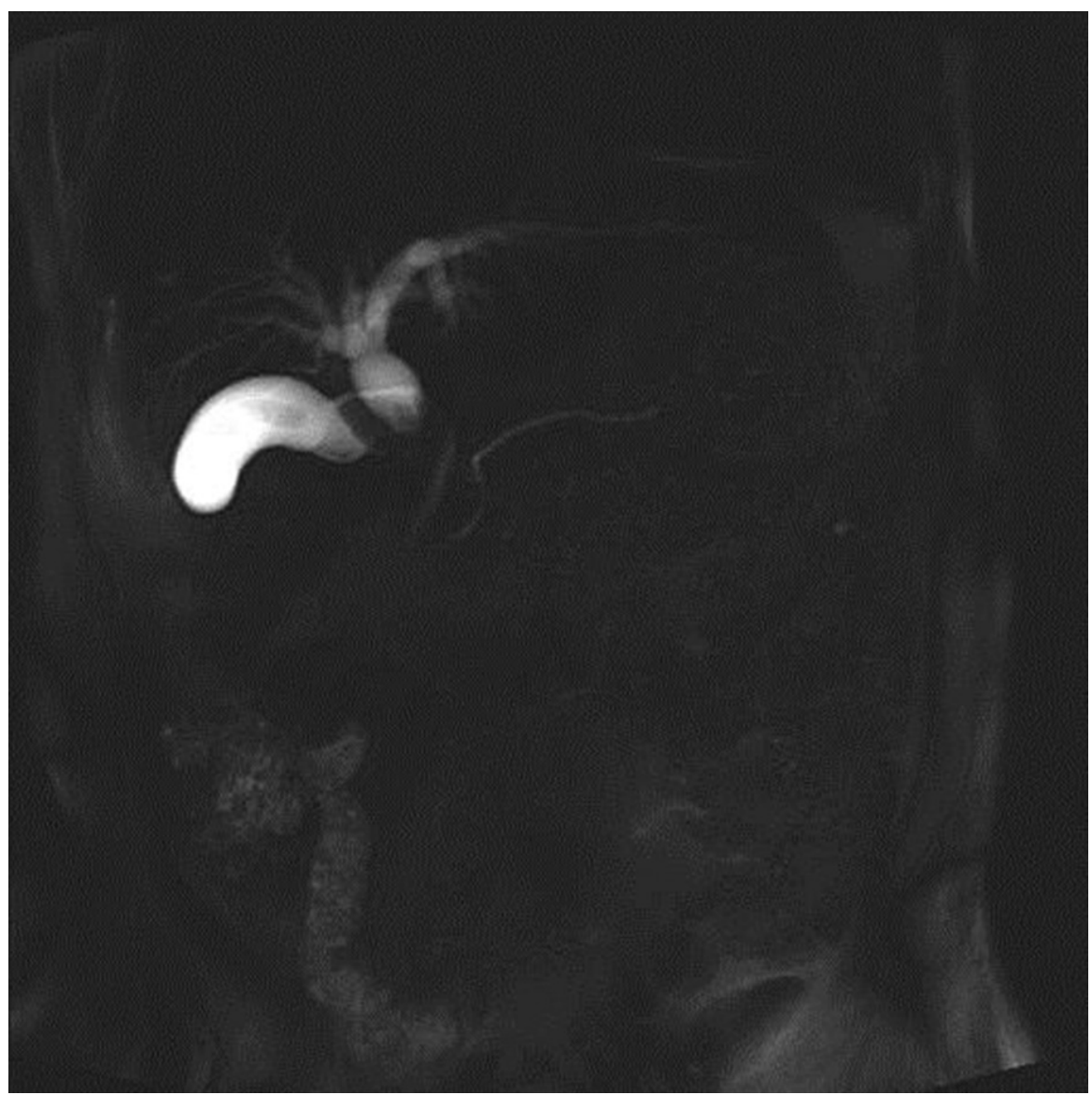

Figure 1: Cholangioresonance showing the filling failure because of the stone located jn the cystic duct measuring 3 centimeters causing common hepatic duct and intrahepatic biliary ducts dilation. Principal pancreatic duct and choledocho are anatomic.

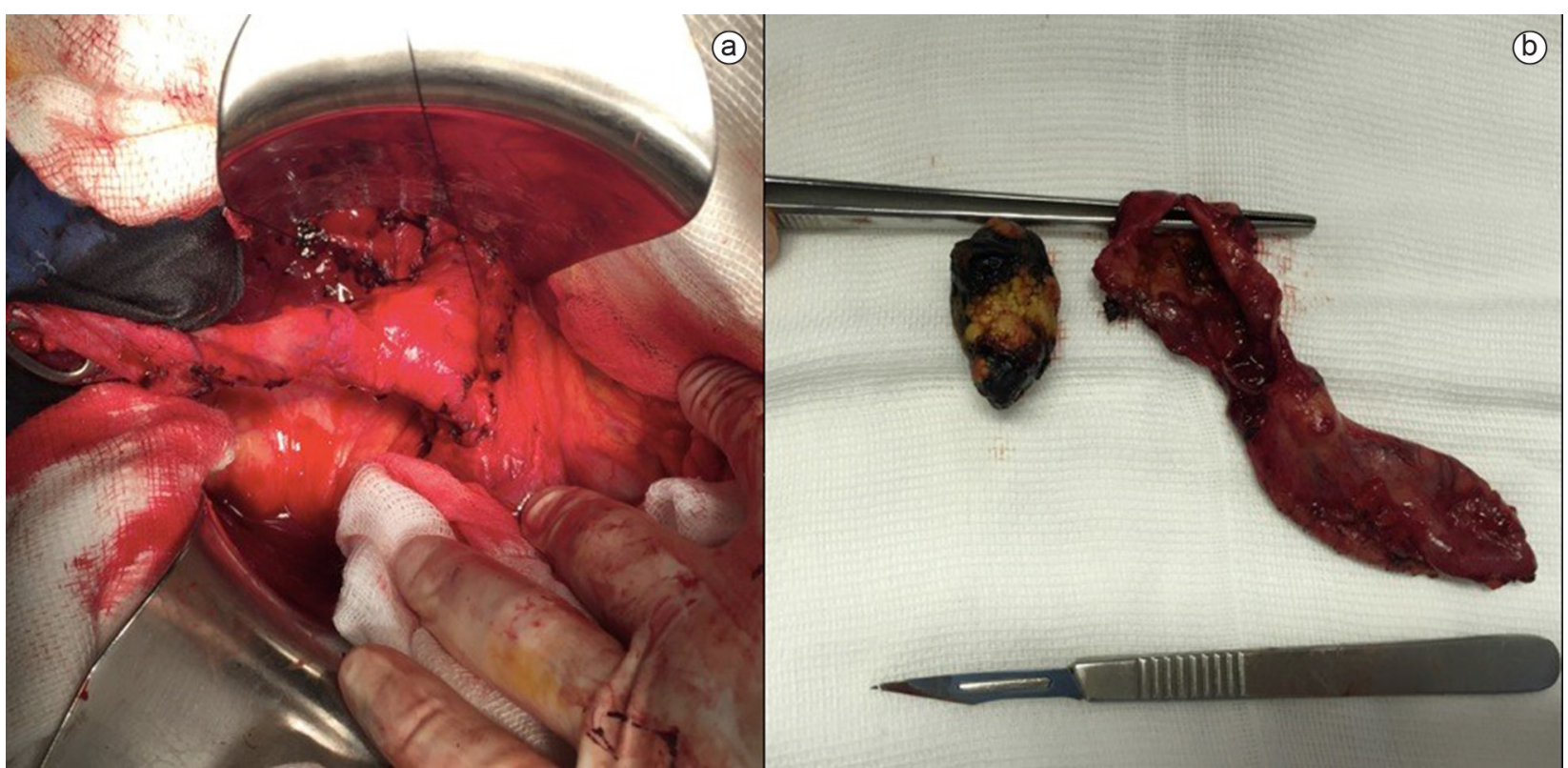

Figure 2: (a) The bloc resection being performed during the procedure; (b) The surgical specimen showing the size of the stone and that it was located exactly in the cystic duct and it demonstrates the bifurcation of the common hepatic and choledocho duct. 


\section{DISCUSSION}

Mirizzi syndrome ${ }^{1,2}$ is a rare complication of cholelithiasis, caused by an extrinsic compression of the common hepatic duct from a stone in the gallbladder neck or in the cystic duct. It occurs in less than $1 \%$ of all cholecystectomies ${ }^{3}$.

According to the modified Csendes classification, there are five different types of Mirizzi syndrome. Type I lesions are those with external compression of the common hepatic duct, while in type II, III and IV a cholecystobiliary fistula is presented, causing erosion of the bile duct, ranging from less than one-third of its circumference, up to two-thirds, and finally with complete destruction of the structure, respectively. Type V, validated by Beltran et al., corresponds to any type of Mirizzi complicated with a cholecystoenteric fistula ${ }^{4}$. The original article describes an incidence of 44 per cent of type III and only 4 per cent of type IV 5 .

Despite the triad of jaundice, fever, and right quadrant pain being present in most patients, it is often not recognized preoperatively. Hence, it may be associated with an increased morbidity, with conversion rate to open surgery of $67 \%{ }^{3}$.

The diagnostic approach usually begins with abdominal ultrasonography, which generally reveals the gallstones and some indications suggestive of biliary obstruction, like dilatation of the biliary system above the level of the gallbladder neck and an abrupt change to a normal width below the level of the stone. Computed Tomography (CT) does not contribute much to sonographic findings with respect to the stone and obstruction, but it is very useful to reveal signs of malignancy, such as enlarged lymph nodes or metastasis ${ }^{6}$.

Cholangiography, either by retrograde endoscopic method or by magnetic resonance is the preferred diagnostic tool because it shows the biliary anatomy more accurately ${ }^{3}$.

A standardized surgical approach has been recommended based on the classification of Mirizzi syndrome. In type I an isolated cholecystectomy is sufficient, while in type II a cholecystectomy accompanied by closure of the fistula, either by suture or by another technique, is preferable. In types III and IV, a bilioenteric anastomosis is required, remaining choledochoplasty as an option for type III cases with a favorable anatomy ${ }^{7}$. Due to the established relationship between Mirizzi syndrome and cholecystoenteric fistulas, fistula repair should be added to the other procedures in patients with type $\mathrm{V}^{4}$.

\section{CONCLUSION}

This case report demonstrates a rare condition of the biliary system, which has to be considered as a differential diagnosis in the presence of jaundice. In addition, it indicates the importance of prepared surgery and radiology services to institute a precise preoperative diagnosis concerning this infrequent condition in the surgery field.

\section{REFERENCES}

1. Mirizzi PL. Sindrome del conducto hepatico. J Int Chir. 1948;8:731-7.

2. Elhanafy E, Atef E, El Nakeeb A, Hamdy E, Elhemaly M, Sultan AM. Mirizzi Syndrome: how it could be a challange. Hepatogastroenterology. 2014;61:1182-6.

3. Erben $Y$, Benavente-Chenhalls LA, Donohue JM, Que FG, Kendrick ML, Reid-Lombardo KM, et al. Diagnosis and treatment of Mirizzi syndrome: 23-year Mayo Clinic experience. J Am Coll Surg. 2011;213:114-9.

4. Beltran MA, Csendes A, Cruces KS. The relationship of Mirizzi syndrome and cholecystoenteric fistula: validation of a modified classification. World J Surg. 2008;32:2237-43.

5. Csendes A, Díaz JC, Burdiles $P$, Maluenda F, Nava O. Mirizzi syndrome and cholecystobiliary fistula: a unifying classification. Br J Surg. 1989;76:1139-43.
6. Becker CD, Hassler H, Terrier F. Preoperative diagnosis of the Mirizzi syndrome: limitations of sonography and computed tomography. AJR Am J Roentgenol. 1984;143:591-6.

7. Baer HU, Matthews JB, Schweizer WP, Gertsch P, Blumgart LH. Management of the Mirizzi syndrome and the surgical implications of cholecystcholedochal fistula. $\mathrm{Br} \mathrm{J}$ Surg. 1990;77:743-5. 